\title{
The influence of surface topography of UV coated and printed cardboard on the print gloss
}

\author{
Authors: Igor Karlović ${ }^{1}$, Dragoljub Novaković ${ }^{1}$, Erzsébet Novotny ${ }^{2}$ \\ ${ }^{1}$ Faculty of technical sciences, Graphic Engineering and Design, Novi Sad, Serbia \\ 2 Állami nyomda printing house, Budapest, Hungary
}

\begin{abstract}
The incident light on the printed surface undergoes through several processes of scattering, absorbtion and reflection depending on the surface topography and structure of the material. The specular part of the surface reflection is commonly attributed as the geometric component of the reflection, and when measured is associated with specular gloss. The diffuse part of the surface reflection contains the chromatic part of the reflection and is commonly calculated through colorimetric values. Using UV coatings as surface enhacement materials which affect the optical properties of coated surfaces and final appearance of the printed product forms new surface topography over the existing one. We have investigated the influence of three different amounts of UV glossy and matte oveprint coating on the measured specular gloss of printed cardboard samples. The different amount of coatings on the printed samples were achived using three different screen stencils of 180 threads $/ \mathrm{cm}, 150$ threads $/ \mathrm{cm}$ and 120 threads/cm thread count. The cardboard samples were analysed with AFM and SEM microscopes to obtain surface topography and roughness values which were evaluated with the measured geometric values speficied as instrumental gloss. The surfaces with a specific amount of UV coatings showed a new formed topography which influences the reflection of light. The changes in topography were evaluated through surface roughness parameters which showed a decline of surface roughness with tht additional ammount of glossy and matte coatings. The obtained and calculated correlations show there is a high correlation between coating ammount and surface roughness change and gloss for the glossy UV coating. The results for the matte UV coatings showed lower correlation for the gloss and surface roughness.
\end{abstract}

Keywords: surface roughness, UV coating, gloss

\section{Introduction}

The surface enhancement of prints as an additional operation is a process where a basic layer of material (plain paper or any other printing substrate), or an already printed surface is oveprinted or coated with a liquid or solid material. The aim of coating these surfaces is the improvement of processing parameters as the drying, physical and chemical protection of prints as well the enhancement of visual appearance and creation of optical effects. The variable factors as the method of application, the type and ammount of applied coating have a strong influence on the process parameters as well the

First received: 23.07.2010.

Accepted: 22.09.2010. measured values, which define the visual appearance values. These interactions and correlations between materials measured from samples can be very useful for determining the suitabillity of reflection models which form the basis for computer simulation of surface enhanced prints. To achieve more accurate reflection model which can be applied for light measurement, it is very important to discuss the different characteristics of surface topography and the compositio of materials because these phyisical values determine the models usability. When an incident light falls on an non ideal surface it is scattered in all directions after the inital surface reflection. In the most general meaning, scattering is the excitation of charged particles by, and the subsequent reradiation of electromagnetic waves. 'The key point is that the re- 
radiated waves are in all directions and the sum of the reradiated waves that emerge at a given spatial position is very much dependent on the physical composition and structure of the material and medium involved' (Hsien Lee, 2005: $\mathrm{p}$ 81.). The manner in which light is reflected by a surface is dependent on, among other factors, the microscopic shape characteristics of the surface. A smooth surface, for instance, may reflect incident light in a single direction, while a rough surface will tend to scatter light in various directions, maybe more in some directions than others. To be able to accurately predict the reflection of incident light, we must have prior knowledge of the microscopic surface irregularities; in other words, we need a model of the surface. All possible surface models may be divided into two broad categories: surfaces with exactly known profiles and surfaces with random irregularities (Nayar and Oren, 1995).

\section{Statement of the problem}

The percepcion of the gloss is usually correalted to the way objects reflect light from their surfaces and is usually perceived indenpedently from the colour appearance; but the underlying colour of the object can influence the perception of the gloss and vice versa. Nevertheless most often the gloss appearance is excluded from the total visual stimulus as it is separated from the colour appearance (Pointier, 2003). To determin preciselly the gloss values it is important to quantify them by an appropriate measurement device. The classical glossmeters are measuring the intensity of the specular reflection of the sample $\left(\mathrm{I}_{\text {sample }}\right)$ relative to some smooth reference standard ( $\left.\mathrm{I}_{\text {reference }}\right)$ for the appropriate measurement angle. The average value of the specular gloss $\mathrm{G}$ can be defined by the following equation:

$$
\mathrm{G}_{\mathrm{s}}=100 \times \mathrm{I}_{\text {sample }} / \mathrm{I}_{\text {reference }}
$$

The total appearance of any object is the combination of its chromatic attributes (colour defined through hue, saturation, lightness) and it's geometrical attributes (gloss, translucency, texture, shape) inside an surrounding where is the object observed. The investigation of the interaction between these optical parameters was and still is in a focus of many research papers. In their investigation Elias et al. (2004) were using a diffuse reflection spectrometry showed that the visual aspect of the pictures with an applied coating are influenced by the difference between refractive indexes of air, coating and ink layer, the changes in the surface topography and the adsorption of the applied coating. The ink layer was defined as a Lambertian diffuse surface. In their previous work (Elias and Simonot, 2004) they found that the refractive index $n_{p}$ is similar to refractive index of the varnish $n_{v}$, and if the layer of the coating is not absorbing the light and there are no internal reflections there would be no changes in the reflection spectrum of the image. Nevertheless some changes were observed and these changes were explained by the changes of the surfaces roughness. In their paper (Klanšek Gunde et al., 2006) have observed the distribution of the particles of a coating material and found a correlation between the distribution on the surface of the samples and thus it's microtopography. In their findings the authors claim that with the addition of thermoreactive substances as fillers and stabilizers there was a change in surface profile of the samples and thus in gloss values. Both parameters of the surface roughness (average roughness and distance between the peaks) have shown correlation to the specular gloss. The coatings with larger particles had smaller amounts of measured instrumental gloss. These finding as well results in research papers by (Järnströma et al., 2008, 2007), (Berns and de la Rie, 2002) and Arney et al (2006) also indicated that the surface roughness of the samples have a significant influence on the measured instrumental gloss. The use of the overprint coating is mainly done for visual enhancement and mechanical protection of the prints. Liquid coatings in which UV coatings are distributed are applied with an anilox roller or through a screen stencil on the surface of the printed samples. With the application of the additional amount of coating it is changing the previous topography of the sample with no coating, and thus influencing the final amount of reflected light and thus the measured print gloss units. The aim of this research was to find correlation with different glossy and matte coating amounts, surface roughness parameters and specular gloss.

\section{Methods and materials}

In the experimental part we have used one commercial glazed cardboard Casino Classic $\left(320 \mathrm{~g} / \mathrm{m}^{2}\right)$ which properties, defnied by the producer, are presented in Table 1.

\section{Table 1. Properties of the used cardboard Casino} Classic

\begin{tabular}{|c|c|}
\hline Substance & $320 \mathrm{~g} / \mathrm{m}^{2}$ \\
\hline Caliper & $300 \mu \mathrm{m}$ \\
\hline Brightness R 457 & $93 \%$ \\
\hline Stiffnes md Minimum value & $2500 \mathrm{mN}$ \\
\hline Smoothness Bekk & 425 \\
\hline
\end{tabular}

The prints were made on Heidelberg Speedmaster SM 52 four colour offset machine, and the coating was made offline on a screen coating machine. The underlying colour over which the coatings were applied were printed using the ISO 12647-2:2004 inking specifications. We have used Sicpa 770 series UV inks and com- 
merical UV glossy and matte coatngs from the Unico 946 series. All the drying procedures as well the other production parameters were kept under control and producer recomended values were used. The coatings was done by using three different screen stencils of 180 threads/cm, 150 threads/cm and 120 threads/cm thread count, which were choosen to apply different amounts of coatings on the prints. The coatings were dryed with the standard UV lamp on the SPS Rhemus screen coating machine. For all the stencils the thread thickness for the was $27 \mu$. The differences in the thread count influence the theoretical transfer amount of coatings which is $6.5 \mathrm{~g} / \mathrm{m}^{2}$ for the 180 thread $/ \mathrm{cm}$ stencil, $9.6 \mathrm{~g} / \mathrm{m}^{2}$ for the 150 thread count stencil and $17.2 \mathrm{~g} / \mathrm{m}^{2}$ for the 120 thread count stencil. For the surface evaluation we have used the Vecco CP-II SPM scanning probe microscope and JEOL 646OLV electrone miscrope. The samples which were determined from the random sampling of prints without production defects and in standard values were evaluated in 3 positions and in 2 directions for all the samples. The AFM scanning area was $80 \mathrm{x}$ $80 \mu \mathrm{m}$ and for the evaluation of the measured results the Image Metrology SPIP software was used. For the surface roughness properties evaluation the following ISO/DIS 25178-2:2010 and ASME B46.1 conforming area profiling parameters were used:

- $\mathrm{S}_{\mathrm{a}}$ Roughness Average [nm]

- $\mathrm{S}_{\mathrm{q}}$ Root Mean Square [nm]

- $S_{\mathrm{v}}$ Max Valley Depth

- $S_{\mathrm{p}}$ Max Peak Height

The roughness average parameter $\mathrm{Sa}$ is the arithmetic average of the absolute values of the measured height deviations from the mean surface taken within the evaluation area and can be expressed for the $\mathrm{Zjk}$ digitalized profile values with the following equation (ASME B46.1):

$$
S a=\frac{1}{M N} \sum_{k=1}^{M} \sum_{j=1}^{N}\left|Z_{j k}\right|
$$

Where $\mathrm{M}$ the number of points per profile and $\mathrm{N}$ is the number of profiles within the sampling area.

The root mean square average of the measured height deviations from the mean surface taken within the evaluation area $\mathrm{Sq}$ can be expressed by the following equation:

$$
S q=\left[\frac{1}{M N} \sum_{k=1}^{M} \sum_{j=1}^{N} Z_{j k}^{2}\right]^{1 / 2}
$$

To observe more precisely the forming of the new surface topography on the prints with the additional amounts of applied coatings, we have also used two additional parameters; the maximum area peak height, $\mathrm{S}_{\mathrm{p}}$ : it represents the maximum height in the evaluation area with respect to the mean surface, while maximum area valley depth, $S_{v}$ is the absolute value of the minimum height in the evaluation area with respect to the mean surface.

The coatings quantity was determined in $\mathrm{g} / \mathrm{m}^{2}$ which is a standard way in the industry to calculate the ammount of coatings on the surface. The values for the used UV coatings were measured on 6 patches of $10 \times 10 \mathrm{~cm}$ samples which were measured with an analytical laboratory scale. The results were obtained from the difference of coated and non coated samples. The instrumental gloss as the parameter for the evaluation of the geometrical part of the reflection. For the gloss measurement the QIP Glossmaster three angle gloss measurement instrument was used. For the instrumental gloss evaluation the specifications stipulated in the ISO 2813 and ASTM D 523 were used. The gloss values were measured on the $100 \%$ tone value patches of the CMYK colours. The statistical parameters were calculated using Statistica 9 software package.

\section{Results}

For all the measured samples we have determined a specific position on the printed sheets were the samples were taken to minize the influence of the coating and printing machine. All the samples which were evaulated and area scanned using the SPIP software a 3D visualization, and the calculation of the surface roughness amplitudal parameters were calculated. The first sample was the printed non coated samples which shall be the starting reference to the glossy and matte coated samples. The scanned surface of the samples are presented in Figure 1. and the averaged measurement data for the surface roughness parameters in Table 2.

As we can observe from Figure 1.a) to g) and values from Table 2. with the application of the UV glossy coating there is decrease in average roughness parameters $\left(\mathrm{S}_{\mathrm{a}}\right.$ and $S_{q}$ ) from the inital value of 169.27 and 242.52 to 38.86 and 46.17 with the lowest quantity of UV glossy coating applied with the 180 threads/cm stencil (7.92 g/ $\mathrm{m}^{2}$ ). The subsequent amount of coatings obtained with the 150 threads $/ \mathrm{cm}$ stencil $\left(11.31 \mathrm{~g} / \mathrm{m}^{2}\right)$ and 120 threads/ $\mathrm{cm}\left(14.96 \mathrm{~g} / \mathrm{m}^{2}\right)$ even more decreased these values respectively to 21.49 and $13.27 \mathrm{~nm}$ for the $\mathrm{S}_{\mathrm{a}}$ and 29.13 and $17.64 \mathrm{~nm}$ for the $\mathrm{S}_{\mathrm{q}}$ parameter. For the matte coating after applying the initial smallest amount of coating with the 180 threads/cm stencil is a noticable increase in average and root mean square roughness of the coated samples. From the inital $\mathrm{S}_{\mathrm{a}}$ value of $169.27 \mathrm{~nm}$ the value raised to $263.95 \mathrm{~nm}$, and subsequently resulted in smaller values similar to glossy coating $(247.06 \mathrm{~nm}$ and $213.02 \mathrm{~nm}$ ). To additionally try to explain this effect we have analysed two additional parameters the $S_{v}$ and $S_{p}$. There is also a noticale decrease between the deepest valley and highest peak values ranging from 1296.79 and $1564.84 \mathrm{~nm}$ to 124.9 and $207.82 \mathrm{~nm}$ for the largest amount of applied glossy coating. The matte coating 

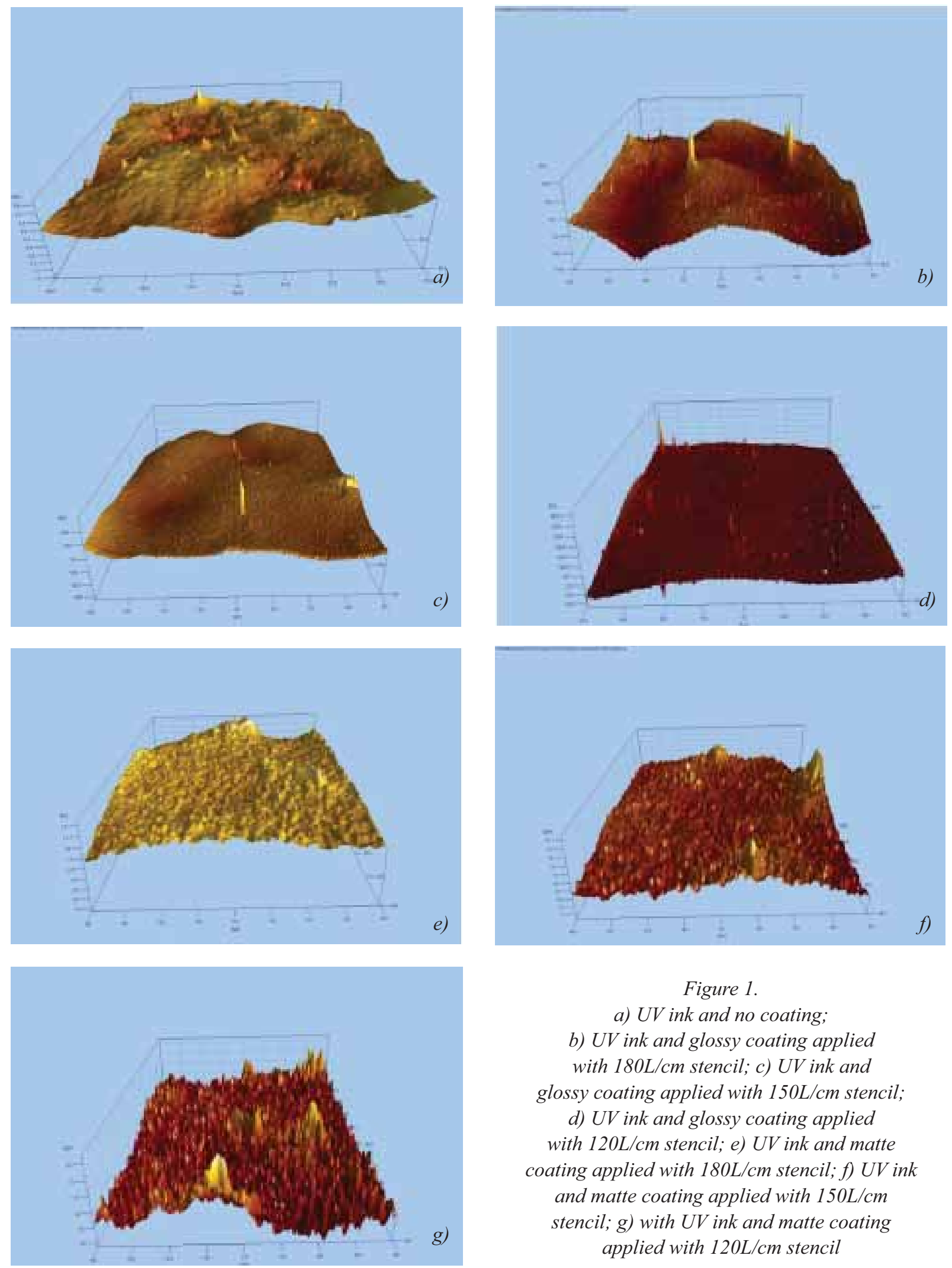

Figure 1.

a) UV ink and no coating;

b) UV ink and glossy coating applied with $180 \mathrm{~L} / \mathrm{cm}$ stencil; c) UV ink and glossy coating applied with $150 \mathrm{~L} / \mathrm{cm}$ stencil;

d) UV ink and glossy coating applied with 120L/cm stencil; e) UV ink and matte coating applied with $180 \mathrm{~L} / \mathrm{cm}$ stencil; f) UV ink and matte coating applied with $150 \mathrm{~L} / \mathrm{cm}$ stencil; g) with UV ink and matte coating applied with $120 \mathrm{~L} / \mathrm{cm}$ stencil

Table 2. The surface roughness parameters averaged values of 6 samples for the cardboard samples

\begin{tabular}{|c|c|c|c|c|c|c|c|}
\hline Parameter & $\begin{array}{c}\text { UV ink } \\
\text { No coating }\end{array}$ & $\begin{array}{c}\text { UV Glossy } \\
180 \mathrm{~L} / \mathrm{cm}\end{array}$ & $\begin{array}{c}\text { UV Glossy } \\
150 \mathrm{~L} / \mathrm{cm}\end{array}$ & $\begin{array}{c}\text { UV Glossy } \\
120 \mathrm{~L} / \mathrm{cm}\end{array}$ & $\begin{array}{c}\text { UV Matte } \\
180 \mathrm{~L} / \mathrm{cm}\end{array}$ & $\begin{array}{c}\text { UV Matte } \\
150 \mathrm{~L} / \mathrm{cm}\end{array}$ & $\begin{array}{c}\text { UV Matte } \\
120 \mathrm{~L} / \mathrm{cm}\end{array}$ \\
\hline Sa [nm] & 169.27 & 38.86 & 21.49 & 13.27 & 263.95 & 247.06 & 213.02 \\
\hline Sq $[\mathrm{nm}]$ & 242.52 & 46.17 & 29.13 & 17.64 & 348.66 & 322.583 & 276.37 \\
\hline Sv $[\mathrm{nm}]$ & 1296.79 & 163.003 & 167.51 & 124.9 & 1766.31 & 1280.44 & 976.69 \\
\hline Sp $[\mathrm{nm}]$ & 1564.84 & 274.170 & 209.612 & 207.82 & 2216.1 & 1596.54 & 1490.29 \\
\hline
\end{tabular}


however first raised the $S_{v}$ and $S_{p}$ parameters with the smallest coating amount of $10.92 \mathrm{~g} / \mathrm{m}^{2}$ (for the $180 \mathrm{~L} /$ $\mathrm{cm}$ stencil) to 1766.31 and $2216.1 \mathrm{~nm}$ respectively. The second amount of UV matte coating yielded the coverage of $10.95 \mathrm{~g} / \mathrm{m}^{2}$ and slightly lower $\mathrm{S}_{\mathrm{v}}$ and $\mathrm{S}_{\mathrm{p}}$ values of 1280.44 and $1596.54 \mathrm{~nm}$. The largest amount of coating gained by using the 120 threads/cm stencil (17.48 $\mathrm{g} / \mathrm{m}^{2}$ ) resulted in the lowest deepest valley and highest peak values of $S_{v}=976.69 \mathrm{~nm}$ and $S_{p}=1490.29 \mathrm{~nm}$ values. The difference between averaged $S_{v}$ values of
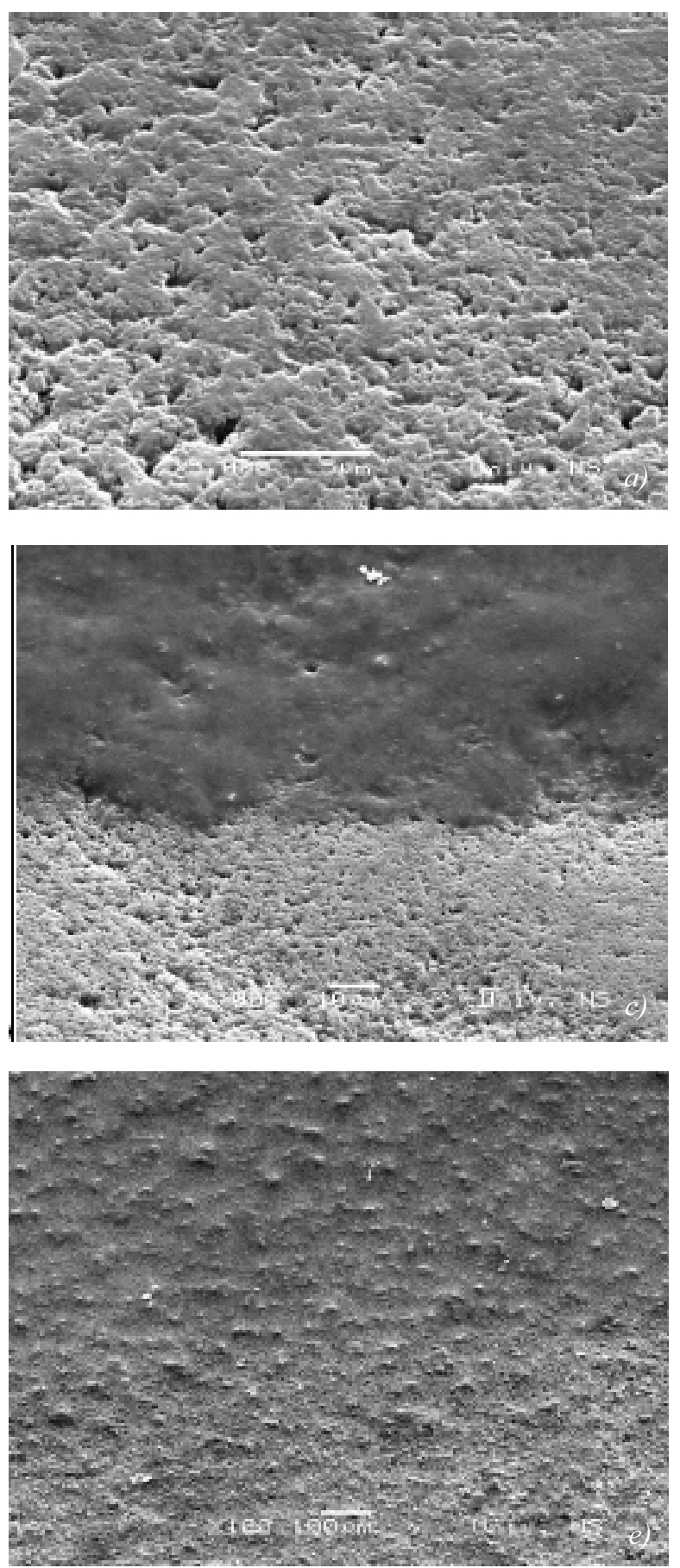

the smallest and largest amount of coating was 789.62 $\mathrm{nm}$, and for the peaks the averaged $\mathrm{S}_{\mathrm{p}}$ values $725.81 \mathrm{~nm}$ which indicate that the valley between the particles was slight more filled with the excessive coating applied on the surface of the prints.

To have a more precise picture of the surface phenomena we have made a quantitative analysis of the surface with a SEM microscope. We have used the JEOL 646OLV electrone miscrope. In Figure 2. we can see the surfaces of cardboard and UV ink on the cardboard
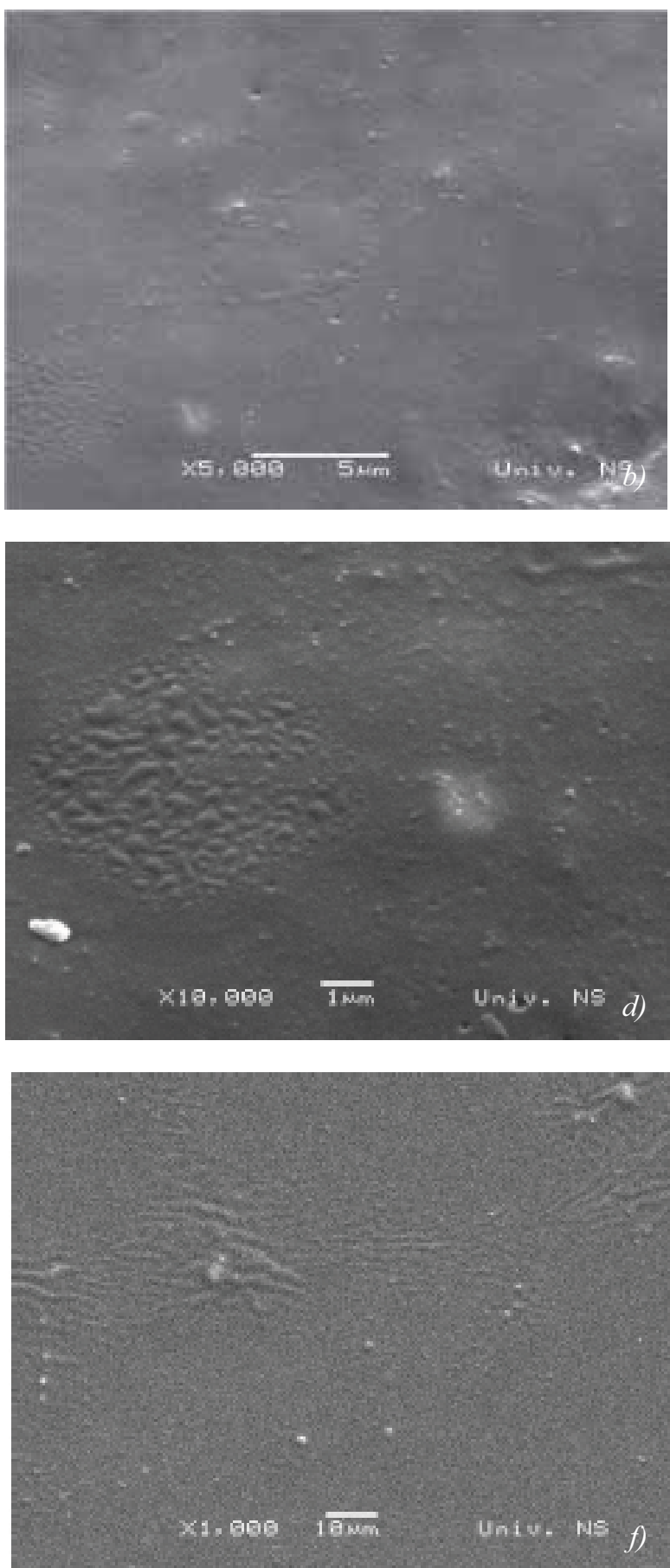

Figure 2. The SEM pictures of the surfaces of a) cardboard with no coating and ink;

b) UV ink; c) the border between ink and plain cardboard surfaces; d) glossy coating applied with the 180 threads/cm stencil; e) matte coating applied with the 180 threads/cm stencil; f) glossy coating applied with the 150 threads/cm stencil 

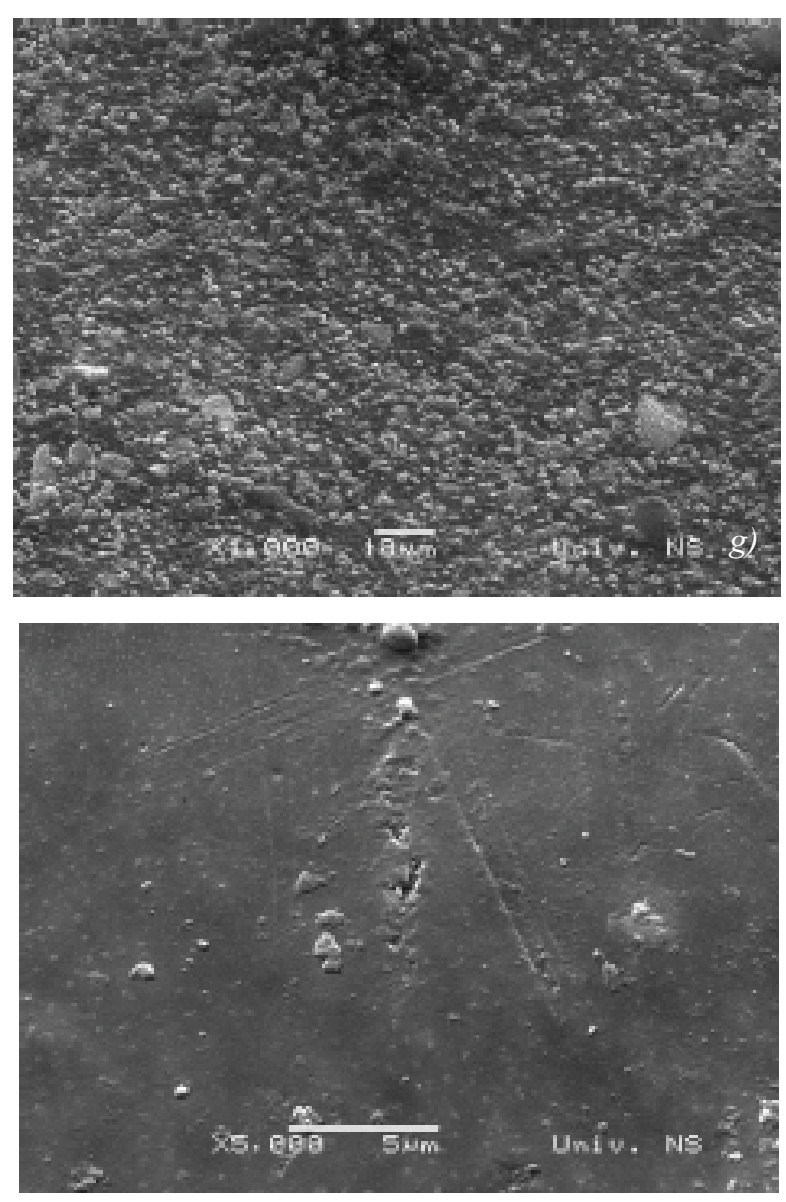

before applying any coating and some examples of the applied coatings. We can observe that with the application of UV ink and UV glossy coatings there is a smoothing effect on the inital rough surface and the ink filss up the valleys on the cardboard surface structure and gives a fairly less rough surface with smaller irregularities. The glossy coating gives a very smooth surface with smaller irregularities and with some spots of coating wrinckling. In these magnifications there are no large visual differences between the surface of different amounts of coatings. With the application of matte coating there is a visible roughning of the inital ink surface and where the matte particles are visible for the lesser to amount so the applied coatings. The largest amount of UV matte coating has a fairly smooth surface where we can see some larger irregularities and a repetitive kind of spots which can be the matting agents which are dipped in the coating. These pictures show the mechanism of the coating leveling and confrom with the previous study made by AFM scanning methodology.

After measuring the surface roughness values we have measured the print gloss values of the coated samples. The results of the print gloss measurements of UV glossy coated samples are presented in Figure 3.

As we can see from Figure 3. for the samples which were not coated we have a gloss range from 44 for the cyan patch to 46.27 for the black patch. The smallest standard deviation had the yellow colour with $\sigma=0.1$,

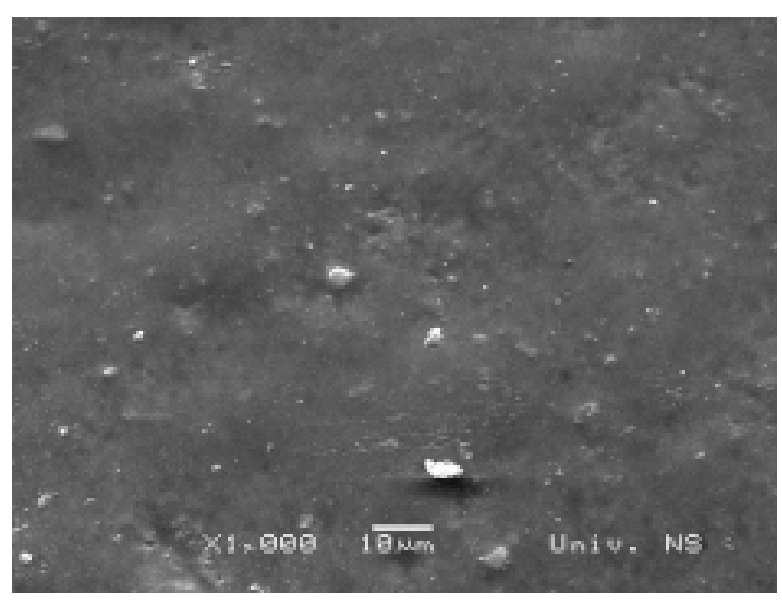

Figure 2. (continued) The SEM pictures of the surfaces of g) matte coating applied with the 150 threads/cm stencil; h) glossy coating applied with the 120 threads $/ \mathrm{cm}$ stencil; i) matte coating applied with the 150 threads/cm stencil;

while the largest standard deviation had the measurement of the magenta samples with $\sigma=0.86$. The print which have been coated with the smallest amount of coating (180 threads/cm) the gloss varied between 46.28 to 51.27 , with the smallest $\sigma=0.36$ for the magenta and $\sigma=1.21$ for the yellow patches measurements. The next amount of coating $\left(11.31 \mathrm{~g} / \mathrm{m}^{2}-150\right.$ threads/ $\mathrm{cm}$ stencil) yielded the range of gloss from 54 for the cyan and 50.53 for the black colour patches. The black patch had also the smallest deviation of $\sigma=0.1$ while the largest calculated value of $\sigma=1.4$ was calculated for the cyan patches. The next applied amount of coating (stencil of 120 threads/cm) resulted in avergaed values of 58.60 for the black colour patch with $\sigma=1.37$ and 55.48 for the yellow colour with $\sigma=0.08$.

After the glossy UV coating we have also measured the print gloss valeus of prints with UV matte coating and the results are presented in Figure 4.

From the results displayed in Figure 4. we can see that the matte coating has a smaller print gloss range but shows some similarity with the glossy UV coatings. The gloss range for the $10.92 \mathrm{~g} / \mathrm{m}^{2}$ coating amount ranged from 13,50 for the yellow and 15,10 for the cyan colour patch. The standard deviation for most of the samples was $\sigma=0.06$. The prints with a slightly larger amount of transfered coating had the gloss range from 19 for magenta and 20.40 for the yellow colour. Magenta and most of the colour patches on which we were measuring the print gloss had the standard deviation value of 


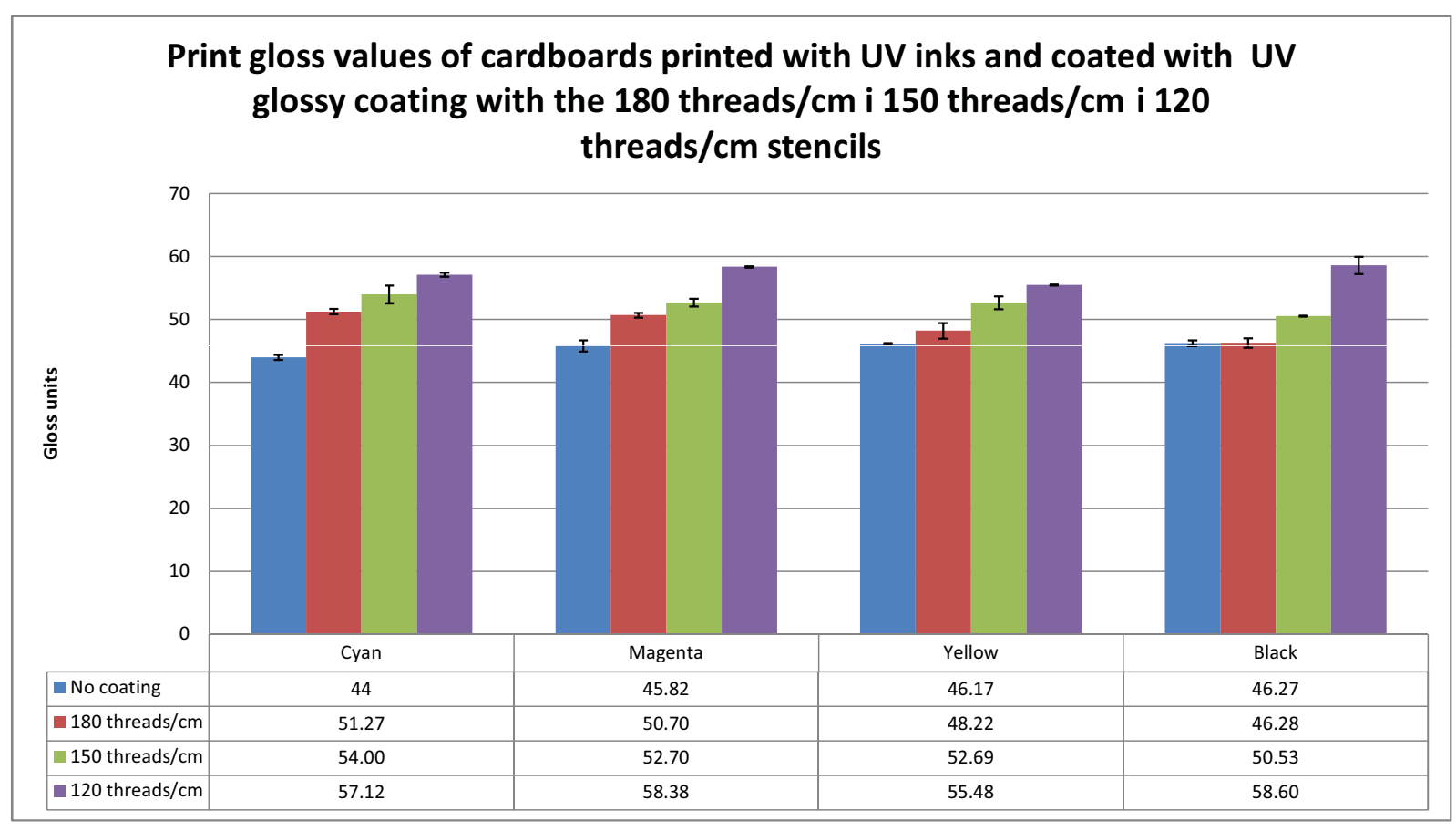

Figure 3. The print gloss values of UV glossy coated samples

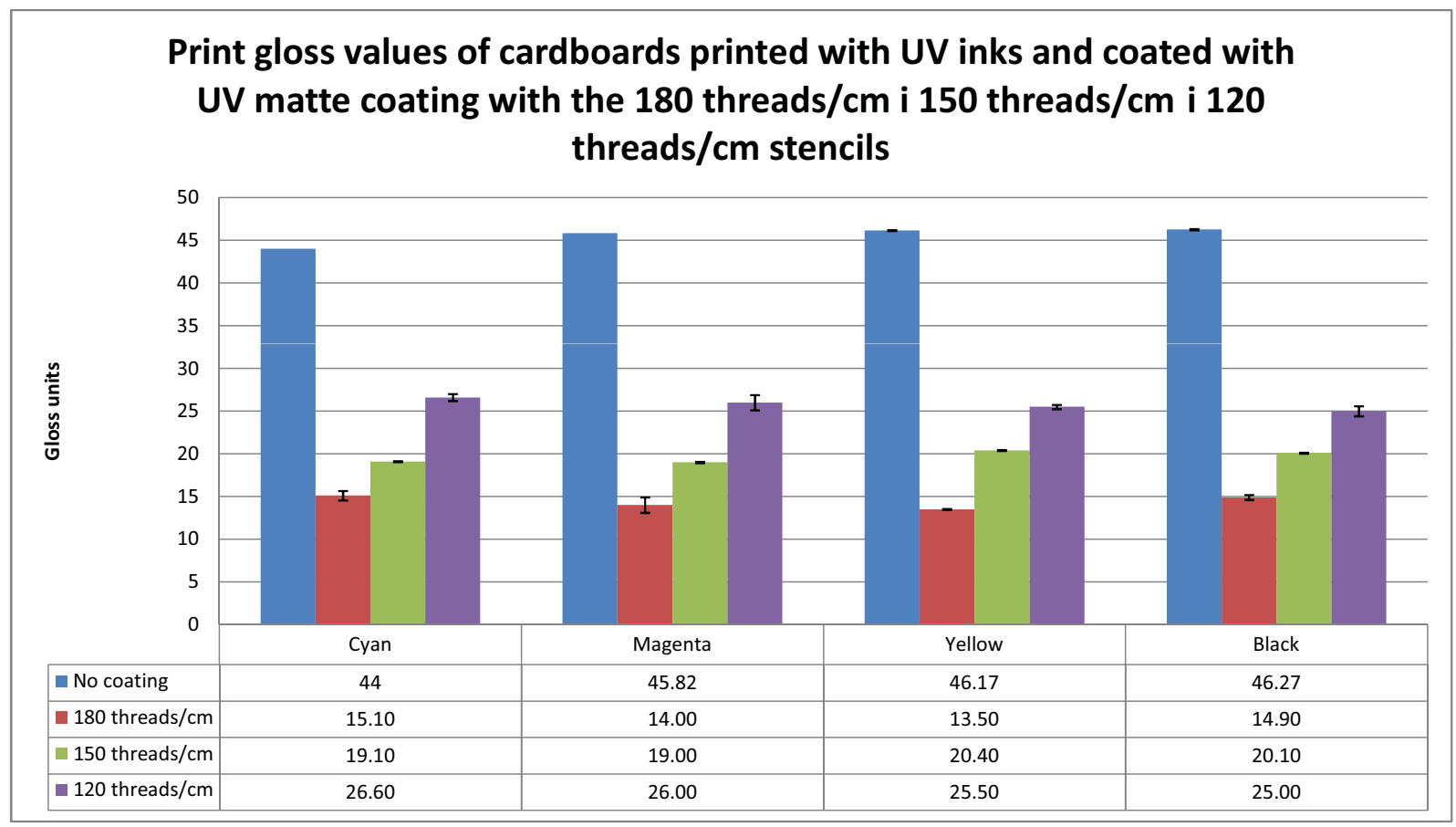

Figure 4. The print gloss values of UV matte coated samples

$\sigma=0.06$. The largest amount of transfered matte coating $\left(17.48 \mathrm{~g} / \mathrm{m}^{2}\right)$ had the largest print gloss values which were ranging from 26.60 for the cyan $\sigma=0.40$ to 25 print gloss units for the black colour with $\sigma=0.58$. With these results we can notice that after the initial decrease of the print gloss units with the smallest amount of UV matte coating, there is an increase with each subsequent coating amount which shows a similar behaviour like the UV glossy coatings on the cardboard. The UV matte coatings had larger transfered amounts on the sheets, this is due to their physical and chemical properties.

\section{Discussion}

After measuring all the values necessary for the analysis we have calculated the correlation factors of the surface roughness, coating quantity and the instrumental measured print gloss. For the correlation calculation we have used the linear fitting model and calculated the appropriate $r$ values. The correlation for the glossy UV coating in terms of avergae surface roughness $\mathrm{S}_{\mathrm{a}}$ in $\mathrm{nm}$, coating quantity in $\mathrm{g} / \mathrm{m}^{2}$, and print gloss is presented in Figure 5. The similar correlation 
between these factors, but for the matte coating are presented in Figure 6.

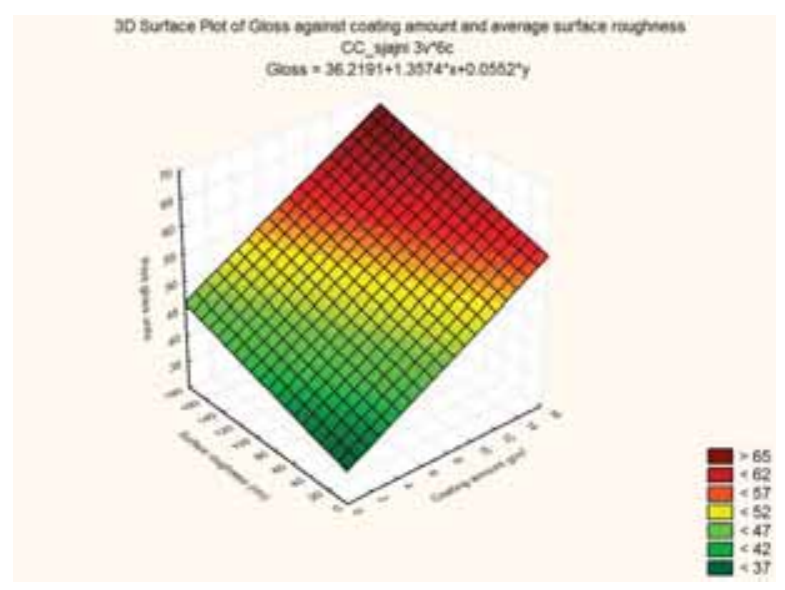

Figure 5. The Correlation between print gloss, surface roughness and coating quantity for the glossy $U V$ coating

As we can observe from the Figure 5. the correlation between the instrumental specular gloss of UV coating and the different amount of the applied coating is $\mathrm{r}=$ 0.96, while the correlation between gloss and surface roughness is defined by the correlation factor $\mathrm{r}=-0.81$. As we can see from the Figure 5 and the calculated factors there is a strong correlation between the coating amount and increase of print gloss, and a smaller linear correlation between the decrease of surface roughness and increase of print gloss. The correlation between the coating amount in $\mathrm{g} / \mathrm{m}^{2}$ and the average surface roughness in $\mathrm{nm}$ was $\mathrm{r}=-0.94$, which indicates that with the application of additional amount of coatings there is a linear decrease of average surface roughness. We have also claculated the correlation factors for the of $S_{v}$ value the maximum valley depth parameter and the maximum peak value $S_{p}$. The linear correlation of $S_{v}$ value to coating quantity was $r=0.90$ and $r=-0.76$, so there is a higher linear correlation between the decrea of valley depth and coating quantity and smaller linear correlation with change of valley depth and gloss. The $S_{p}$ value yielded the same correlation values with the gloss and coating amount as the $S_{v}$ parameter. This indicates there is a similar change in these parameters in the process of applying additional amounts of UV glossy coatings. The correlation between matte coating and the average surface roughness is presented in Figure 6.

As can be seen from Figure 6. there is an increase in print gloss values after the application of additional amount of coating. After calculating between the variable factors we have determined that for the linear fitting between the print gloss and coating quantity the $r$ value is $r=-0.67$, while the correlation between the gloss and surface roughness is $r=-0.97$. The relationship between the surface roughness and coating quantity resulted in just $r=0.52$. The lower linear correlation

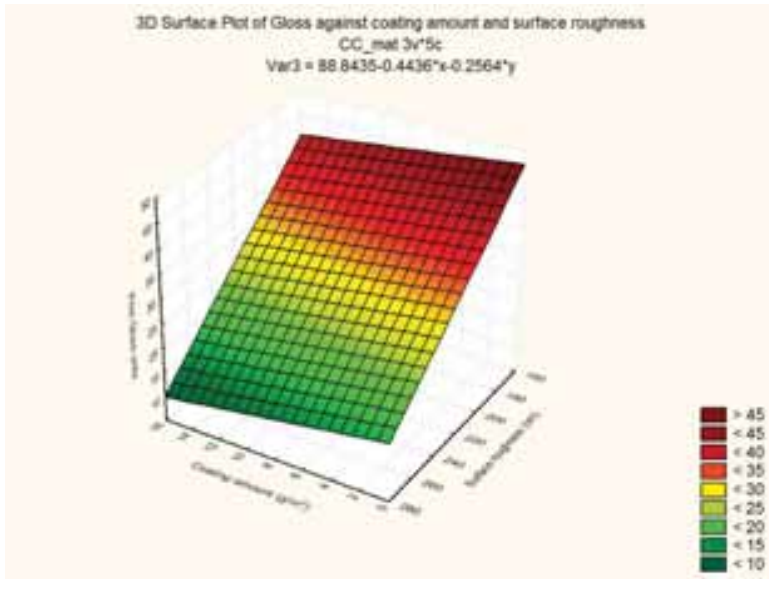

Figure 6. The Correlation between print gloss, surface roughness and coating quantity for the matte $U V$ coating

values are calculated because after the inital decreae of gloss, the subsequent larger amount of coating resulted in higher measure instrumental gloss. If we exclude the inital values with no coating applied and calculate the relationship between these values only with coated samples we get $\mathrm{r}=0.98$ for the gloss and coating correlation, $r=-0.94$ for the gloss and avergae surface roughness and $\mathrm{r}=-0.98$ for the coating quantity and surface roughness. With these calculated values we can observe that the higher amount of the transfered UV matte coating results in increase of print gloss and with the increase of gloss there is a decrease of the avergae surface roughness. The values for the $S_{v}$ parameter were $r=0.55$ for the coating amount and valley depth and $r=-0.98$ for the valley depth and print gloss values. The $\mathrm{r}$ factors for the $\mathrm{S}_{\mathrm{p}}$ parameters yielded in $\mathrm{r}=0.66$ (coating amount and peak height) and $r=-0.99$ for the peak height and print gloss values.

\section{Conclusion}

In these paper we have researched the influence of type and amount of UV coatings on the printed cardboard in terms of instrumental measured print gloss. After inspecting the surfaces of the sample with AFM and SEM microscopy we have observed changes in the initial surface of printed cardboard where the glossy UV coating has smoothened the surface while the UV matte coating gave a rougher surface. The measurement of print gloss showed that the amount of gloss is much higher on the glossy UV coated samples in comparisment to the matte coated samples. In both cases the larger amount of coating resulted in higher print gloss values then the previous smaller amount. While there is a linear rise for the glossy coated UV samples from the initial printed but not coated samples to the highest amount of applyied coating, the UV matte coating first decreases the print gloss but with the additional amount of coating it in- 
creases the print gloss which with the heighest amount of coating applied with the 120 threads/cm screen stencil is measuring half values of the non coated printing inks. The changes in print gloss, average surface roughness and other calculated surface roughness parameters show a somewhat linear type of correlation between coating amount and print gloss, and smaller but still indicative values for the surface roughness parameters and gloss. These values show that surface roughness is not the singular factor affecting the print gloss (but nevertheless a important one), but also the chemical and psyhical properties of the applyed coating. These results can be used for software simulations, production optimization and reduction in coating usage which enables better enviromental protection and can influence the choice of the coating quantity, type and method of application in strive of achieving good quality reproduction and standardized production.

\section{References}

1. Arney, J. S., Ye, L., Banach, S. (2006) Interpretation of Gloss Meter Measurements. Journal of Imaging Science and Technology, 50 (6), pp. 567-571.

2. Berns, R., de la Rie, R.E. (2002) The relative importance of surface roughness and refractive index in the effects of varnishes on the appearance of paintings. In: R. Vontobel (ed.) ICOM-CC Triennial Meeting Preprints, 22-27 September 2002, Rio de Jeneiro, Brasil. London, James \& James, Science Publishers. pp. 211-216.

3. Elias, M., Simonot, L. (2004) Bi-directional reflectance of a varnished painting, Part 1: Influence of the refractive indices without using the approximations of Saunderson correction - exact computation. $O p$ tics Communications, 231 (1-6), 17-24.

4. Järnströma, J., Ihalainenb, P., Backfolkc, K., Peltonenb, J. (2008) Roughness of pigment coatings and its infuence on gloss. Applied Surface Science, 254 (18), pp. 5741-5749

5. Järnström, J., Peltonen, J., Sinervo, L., Toivakka, M., (2007) Topography and gloss of precipitated calcium carbonate coating layers on a model substrate. Tappi Journal, 6(5), p. 23.

6. Klanjšek, G. M., Kunaver, M., Čekada, M. (2006) The influence of surface texture parameters on specular gloss of powder coatings. V: CIE expert symposium on visual appearance, 19-20 October 2006, Paris, France. [Online] 29. Avaliable from: http:// www.mnhn.fr/cievisualappearance/Abstract $\% 20$ book.pdf [Accessed 5th May 2010].

7. Simonot, L., Elias, M. (2004) Color change due to a varnish layer. Color Research and application, 29 (3), pp.196-204

8. Lee, H. C. (2005) Introduction to Color Imaging Science. Cambridge, Cambridge University Press
9. Nayar, S. K., Oren, M. (1995) Visual appearance of matte surfaces. Science, 267, pp. 1153-1156

10. Pointer, M. (2003) Measuring visual appearance - a framework for the future, Project 2.3 Measurement of Appearance. NPL REPORT: COAM 19, National Physical Laboratory Queens Road, Teddington, Middlesex 